\title{
Ensaio didático sobre os conjuntos de Julia e de Mandelbrot
}

\author{
Colaboração Matemática Aberta ${ }^{1}$
}

8 de Maio de 2021

\begin{abstract}
Resumo
Definimos matematicamente de forma sucinta os conjuntos de Julia e de Mandelbrot.
\end{abstract}

palavras-chave: conjunto de Julia, Mandelbrot, fractal, caos, sistema dinâmico

A versão mais atualizada deste artigo está disponível em https://osf.io/fkvrt/download

\section{Introdução}

1. Apresentamos aqui o mínimo necessário para que os leitores possam se vislumbrar com os fractais produzidos pelos conjuntos de Julia e de Mandelbrot.

2. Mais detalhes podem ser encontrados neste excelente livro introdutório [1].

${ }^{1}$ Todos os autores com suas afiliações aparecem no final deste artigo. 


\section{Função, condição inicial e órbita}

3. Considere a função $f(x)=x^{2}$ para $x \in \mathbb{R}$.

4. Seja $x_{0}$ a condição inicial.

5. Considere a seguinte notação:

$$
f^{1}:=f\left(x_{0}\right), \quad f^{2}:=f\left(f^{1}\right), \quad f^{3}:=f\left(f^{2}\right), \quad \ldots
$$

6. Faremos iterações de $f(x)$, o que significa calcular $f^{1}, f^{2}, f^{3}, \ldots$

7. A órbita de $x_{0}$ é dada por

$$
x_{0} \longrightarrow f^{1} \longrightarrow f^{2} \longrightarrow f^{3} \longrightarrow \cdots
$$

\section{Função complexa}

8. Considere a seguinte função $g: \mathbb{C} \rightarrow \mathbb{C}$ dada por

$$
g(z)=z^{2}+c
$$

$\operatorname{com} z, c \in \mathbb{C}$.

\section{Conjunto de Julia para a família quadrática}

9. Considere as condições iniciais $z_{i} \in \mathbb{C}$, sendo $i \in \mathbb{N}=\{1,2,3, \ldots\}$.

10. O conjunto de Julia é definido como sendo

$$
J=\left\{z_{i} \mid f^{n}<\infty, n \rightarrow \infty\right\} .
$$

11. Em palavras, $J$ é formado por todas as condições iniciais $z_{0}$ tal que sua órbita é finita. 


\section{Conectado ou desconectado}

12. O conjunto de Julia é dito conexo (desconexo) quando seus pontos estão conectados (desconectados).

13. Pontos desconectados não formam um único objeto contínuo.

\section{Conjunto de Mandelbrot}

14. O conjunto de Mandelbrot é definido como

$$
\mathcal{M}=\left\{z_{0} \mid J\left(z_{0}\right) \text { é conexo }\right\} .
$$

15. Assim, $\mathcal{M}$ inclui apenas os conjuntos de Julia que são conexos.

\section{Exemplos}

16. $[1-4]$

17. As cores que aparecem nos exemplos tem a ver com a taxa com que as condições iniciais vão para o infinito, isso está muito bem explicado em [1].

\section{Considerações Finais}

18. As definições matemáticas para os conjuntos de Julia e Mandelbrot são incrivelmente simples e eles geram padrões igualmente incríveis.

\section{Ciência Aberta}

$\mathrm{O}$ arquivo latex para este artigo, juntamente com outros arquivos suplementares, estão disponíveis em [5]. Seja coautor(a) deste artigo, envie sua contribuição para mplobo@uft. edu.br. 


\section{Consentimento}

19. O autor concorda com [6].

\section{Como citar este artigo?}

20. [7]

\section{Referências}

[1] Feldman, David P. Chaos and fractals: an elementary introduction. Oxford University Press, 2012. http://books.google.com/books?vid=exnWM_ZHKOMC

[2] Lesmoir-Gordon, Nigel, ed. The Colours of Infinity: The Beauty and Power of Fractals. Springer Science \& Business Media, 2010. http://books.google.com/books?vid=ah0e6w3RnP4C

[3] Wikipedia. "Julia set."

https://en.wikipedia.org/wiki/Julia_set

[4] Wikipedia. "Mandelbrot set."

https://en.wikipedia.org/wiki/Mandelbrot_set

[5] Lobo, Matheus P. "Open Journal of Mathematics and Physics (OJMP)." OSF, 21 Apr. 2020.

https://doi.org/10.17605/osf .io/6hzyp

[6] Lobo, Matheus P. "Simple Guidelines for Authors: Open Journal of Mathematics and Physics." OSF Preprints, 15 Nov. 2019.

https://doi.org/10.31219/osf.io/fk836

[7] Lobo, Matheus P. "Ensaio Didático Sobre Os Conjuntos De Julia E De Mandelbrot." OSF Preprints, 7 May 2021. https://doi.org/10.31219/osf .io/fkvrt 


\section{Colaboração Matemática Aberta}

Matheus Pereira Lobo (autor principal, mplobo@uft.edu.br) $)^{1,2}$ https://orcid.org/0000-0003-4554-1372

${ }^{1}$ Universidade Federal do Tocantins (Brasil)

${ }^{2}$ Universidade Aberta (UAb, Portugal) 